\title{
Impacto y Aprovechamiento de las Tecnologías de la Información y las Comunicaciones en la Educación Superior
}

\author{
María F. Alcibar ${ }^{1 *}$, Anderson Monroy ${ }^{1}$, Martha Jiménez ${ }^{2}$ \\ (1) Instituto Politécnico Nacional, Unidad Profesional Interdisciplinaria de Ingeniería y Ciencias Sociales y \\ Administrativas (UPIICSA). Av. Té 950, Granjas de México, 08400, Ciudad de México, México. \\ (e-mail: fernandaalhe@gmail.com) \\ (2) Instituto Politécnico Nacional, Unidad Profesional Interdisciplinaria de Ingeniería y Ciencias Sociales y \\ Administrativas (UPIICSA), Sección de Estudios de Posgrado e Investigación. Av. Té 950, Granjas de México, \\ 08400, Ciudad de México, México.
}

${ }^{*}$ Autor a quien debe ser dirigida la correspondencia

Recibido Ene. 29, 2018; Aceptado Abr. 10, 2018; Versión final Jun. 13, 2018, Publicado Oct. 2018

\section{Resumen}

Se analiza y discute el uso de las tecnologías de la información y las comunicaciones (TIC) y su impacto en la educación superior, y en particular en las actividades académicas como la realización de tareas escolares elaboradas con la búsqueda de información en la Internet. Se aplicó una encuesta semiestructurada sobre el uso de las TIC para el desarrollo de actividades académicas en el Instituto Politécnico Nacional. Se pudo observar que los estudiantes de dicha institución usan diversas herramientas informáticas por lo menos una vez a la semana como medio de consulta para la realización de tareas escolares. Se concluye que aspectos como la computadora, la forma amigable y divertida de hacer las tareas por internet influyen de forma positiva en el aprendizaje.

Palabras clave: TIC; educación superior; redes sociales; herramientas informáticas; tareas escolares

\section{Impact and Use of Information and Communication Technologies in Higher Education}

\begin{abstract}
The use of communication and information technologies (ICT) and their impact on higher education are analyzed and discussed. In particular activities such as homework with the use of information provided by the Internet. A semi structured survey was applied on the use of ICT for the development of academic activities at the National Polytechnic Institute within the Interdisciplinary Professional Unit of Engineering and Social and Administrative Sciences It was observed that the students of this Institution use various computer tools at least once a week as a means of consultation for the completion of school tasks. It is concluded that aspects such as the computer, the friendly and entertaining way of doing tasks online, have a positive influence on learning.
\end{abstract}




\section{INTRODUCCIÓN}

Es evidente que las Tecnologías de información y Comunicación (TIC) han generado un gran impacto en todos los ámbitos de la sociedad, es por ello que la presente investigación aborda el tema de TIC dentro de la educación superior y el efecto que tiene en México. El estudio de las TIC es de gran importancia pues permite la transferencia del conocimiento, del auto aprendizaje y la comprensión (Khan y Markauskaite, 2017), así como el desarrollo de nuevas competencias y habilidades que permiten a los alumnos aumentar sus conocimientos adquiridos dentro de las aulas. Hoy en día, gracias a la tecnología que se ha desarrollado paulatinamente y su utilización en distintos ámbitos de nuestras vidas, ha permitido la posibilidad de favorecer y facilitar distintos procesos; a través de los años se ha introducido en la educación el uso de las TIC con una mejora continua en los procesos de enseñanza aprendizaje, y se ha incorporado de diversas formas para mejorar los procesos de aprendizaje y enseñanza de los estudiantes, para que dichos los alumnos a mediano plazo se incorporen al mercado laboral y sean un personal que enfrente el mundo competitivo.

En los últimos años, de acuerdo a la UNESCO (2014), América Latina es uno de los lugares más proactivos del mundo con relación a la integración de las TIC en la educación que permite la inclusión social, la democratización, así como la reducción de la brecha digital existente; así mismo menciona distintas políticas de integración de las TIC en la educación entre los que se encuentran los laboratorios donde los equipos son escasos, las aulas móviles y el modelo 1 a 1 donde se proveen dispositivos a cada persona para su uso en las aulas con el fin de reducir la brecha digital y la deserción escolar. De igual manera la UNICEF (2013) menciona que la incorporación de las TIC permite que los jóvenes adquieran habilidades y competencias reduciendo así la brecha digital entre grupos sociales, mejorando la educación y maximizando los logros académicos.

Para el caso particular de México, la UNICEF (2013) menciona los programas realizados en el gobierno mexicano resaltando la creación de portales educativos y contenidos digitales no solamente enfocados a los alumnos, sino también a docentes, y otros agentes educativos. Cabe añadir, que el gobierno mexicano señala en el Plan Nacional de Desarrollo algunas estrategias para lograr una educación de calidad y se menciona que hoy en día al tener a la mano a una gran cantidad de información debe de incrementarse la inversión en tecnología, así como la vinculación entre universidades, centros de investigación y el sector privado (Federal, 2013). A través del programa "México conectado" de acuerdo a la Secretaría de Comunicaciones y Transporte (SCT) (2017) se menciona que se ofrece conexión a internet de forma gratuita en distintos lugares públicos como lo son universidades, centros de investigación y bibliotecas permitiendo así el intercambio de información de forma rápida. Actualmente la SCT se encuentra trabajando en distintos programas que permite a la población en general tener acceso a internet como lo garantiza el artículo sexto de la Constitución Política de los Estados Unidos Mexicanos sobre el acceso a las TIC y telecomunicaciones (Cámara de Diputados, 2017).

En general, la enseñanza universitaria se desarrolla a través de cátedras presenciales que presenta los contenidos de las distintas asignaturas que se tienen y al finalizar, suele aplicarse evaluaciones que permiten observar el grado del aprendizaje adquirido por los alumnos (Ausín et al., 2016), sin embargo hoy en día gracias a la incorporación de las TIC se han cambiado las formas de enseñanza, lo que quiere decir que el uso de la tecnología en las universidades no solo es un hecho sino también un desafío tanto para los alumnos como para los profesores, pues hay que reconfigurar la tecnología con la práctica en el aula y las relaciones de poder dentro de las aulas, pues el profesor pierde el poder y los alumnos se sienten más cómodos con esta situación (Flavin, 2017). Así mismo, existen diversos programas educativos que permiten a los alumnos tomar clases en línea que generan nuevas oportunidades para las personas que no tienen la posibilidad de asistir a un aula, volviéndose así estudiantes más autónomos en el proceso de aprendizaje.

En consecuencia se puede observar que el uso de las TIC tiene un papel importante en el ámbito educativo, así mismo el uso efectivo de las TIC permite a los países en desarrollo aumentar el conocimiento en el capital humano, un ejemplo de esto es en la Universidad de arabia Saudita, donde los alumnos tienen interés en la creación de conocimiento y discusión en línea así como el uso compartido de archivos e intercambio por internet a través de redes sociales, blogs y wiki; que no solamente son herramientas que tienen gran impacto en Arabia sino también en Italia donde las principales herramientas utilizadas en el entorno académico son YouTube y wiki (Eid y Al-Jabri, 2016; Manca y Ranieri, 2016). En México se han seguido pautas para la creación de programas sociales que permitan el uso de herramientas tecnológicas dentro de las universidades permitiendo así, generar cambios importantes en los procesos de aprendizaje y enseñanza.

La presente investigación se llevó a cabo con el objetivo de analizar el aprovechamiento de las TIC y su impacto que tiene en la educación superior, en las actividades académicas como la realización de tareas escolares elaboradas con la búsqueda de información en el internet, para ello, se realizó una investigación descriptiva y cuantitativa dentro de la Unidad Profesional Interdisciplinaria de Ingeniería y Ciencias Sociales y Administrativas (UPIICSA) perteneciente al Instituto Politécnico Nacional, debido a que esta escuela es de 
carácter interdisciplinario, pues tiene programas de estudio de ingeniería y del área social; lo cual permite conocer el uso de las herramientas informáticas en distintas áreas de conocimiento, y el impacto que puede tener sobre las mismas, se aplicó una encuesta semiestructurada; con dicho estudio se pretende conocer el uso que los alumnos hacen de las diferentes TIC a las que tienen acceso.

\section{OTROS ANTECEDENTES}

Hay otros antecedentes sobre el tema que es necesario resumir y contextualizar para este trabajo que se presenta: las TIC y la educación superior, el uso del internet y su influencia en el rendimiento académico son de especial interés y se discuten en lo que sigue.

\section{Las TIC y la Educación Superior}

En consecuencia, de la importancia que tienen las TIC en la educación, se ha provocado que los profesores, alumnos e instituciones en general reajusten los procesos con el fin de transformarlos e innovarlos. Por otro lado, las TIC ayuda a los estudiantes a desarrollar distintas competencias que pueden ser utilizadas dentro y fuera del aula. Asimismo, la presencia de las TIC en la formación universitaria favorece los procesos de enseñanza y aprendizaje a profesores y estudiantes, además de mantener un aprendizaje abierto, continuo y flexible al romper las barreras de tiempo y lugar con la posibilidad de dar paso a nuevos criterios y conocimientos que no solamente tienen impacto dentro de la educación, sino también en el ámbito laboral (Garcés et al., 2016).

El uso de dispositivos móviles para el aprendizaje motiva a los alumnos a desarrollar la exploración, el trabajo en equipo, el pensamiento crítico y reflexivo (Basantes et al., 2017), es por ello que la afinidad creada entre las TIC y los estudiantes universitarios los ha llevado a adquirir distintos dispositivos tecnológicos, a capacitarse y a implementarlos en sus distintas actividades dentro de la escuela y su vida profesional (Casillas et al., 2016).

La escuela digital basada en el uso de tabletas con acceso a internet, pero sin telefonía móvil, es un éxito ya que se aumenta considerablemente la calidad del aprendizaje individual y colectivo de la población estudiantil; pues mejora la forma de aprendizaje y se tiene un nuevo conocimiento de forma, esto indica que se puede aprender sin asistir a clases (Witteveen et al., 2017). Sin embargo, hay que poner especial atención en el contenido atractivo y las necesidades de los estudiantes al usar las tabletas electrónicas, pues pueden ser inútiles, lo cual llevaría al fracaso y afectar así la calidad educativa (Pruet et al., 2016). Dicho lo anterior, los alumnos deben ser capaces de adquirir distintas habilidades que les permitan lograr un aprendizaje significativo al tener a la mano distintos tipos de materiales tanto informativos como visuales que hará personas más autodidactas, capaces de aplicar el pensamiento crítico y trabajar en equipo.

En la educación superior el uso de las aulas virtuales son tecnologías eficientes que incluyen aspectos de multimedia con audio y video asíncrono y síncrono, que han demostrado ser efectivas pues incluyen notas de los profesores, además de crear espacios de foros que los alumnos pueden discutir sobre temas vistos en clase; además le dan vitalidad al aula virtual (Anshari et al., 2015; Caputi y Garrido, 2015; Trust, 2017; Vicent et al., 2015 y Yamashita, 2017).

La comunicación grupal es importante para potencializar la educación superior y esto se logra con el uso de las TIC y del Internet; es por esto que los profesores deben incluir a las TIC en sus proyectos académicos y crear foros virtuales de discusión, mismos que deben estar reformulados para lograr altos niveles de logros académicos; asimismo los profesores deben seleccionar de forma coherente las herramientas de aprendizaje de sus estudiantes acorde a la ejecución de los proyectos formativos de sus alumnos, pues para los profesores es un trabajo difícil pues tienen que enfrentar diversas situaciones de resistencia al cambio tecnológico y afrontarlas al nuevo tipo de sociedad tecnológica (Fainholc, 2008).

La importancia del uso del internet entre los estudiantes en la educación superior, se ha vuelto una herramienta indispensable para la comunicación y acceso ilimitada de información, teniendo así distintas herramientas y fuentes de estudios, así mismo el poseer una computadora, es un requisito indispensable para la educación superior (Linne, 2015).

\section{Las TIC y el rendimiento académico en la educación superior}

Cuando se incorporan distintas herramientas tecnológicas dentro de la educación, los alumnos tienen la capacidad de aprender de diferentes maneras y a ritmos desiguales, sin embargo, el papel del rendimiento académico dentro de los estudios es, aun así, una forma de medir los procesos de aprendizaje de los alumnos de acuerdo a los estándares que se establecen. Sin duda alguna, el rendimiento académico es un patrón utilizado para medir el aprendizaje del alumnado de las distintas asignaturas que permite a los docentes 
observar el conocimiento adquirido y su aplicación, que de acuerdo con Prieto-Velasco y Fuentes-Luque (2016) los estudiantes que utilizan TIC presentan una actitud positiva por la interacción entre los miembros además que el rendimiento académico mostró una mejora significativa.

La incorporación de las TIC en la formación universitaria, revela cambios en el proceso de formación e incremento en el rendimiento académico, viendo resultados en la adquisición de habilidades y actitudes que contribuyen en mejorar el futuro laboral (Faúndez, 2017); además, permite alcanzar una constante innovación dentro de las aulas escolares al tener una ampliación de entornos de aprendizaje y una diversificación de los métodos de trabajo. Asimismo, las aulas virtuales requieren de técnicas de enseñanza innovadoras para ayudar a los profesores en la mejora de aspectos visuales en las cátedras a través del uso de herramientas y software libre que los alumnos utilizan diariamente de forma natural, para lograr así aumentar el rendimiento escolar y propiciar su crecimiento personal (Vahtivuori-Hänninen y Kynäslahti, 2016; Faúndez et al., 2017; Thomas et al., 2017).

Mientras que los docentes utilizan las TIC como una oportunidad para la creación de contenido y conocimiento como presentaciones o videos educativos con audio para compartir con el alumnado y los puedan ver en su casa, reforzando sus conocimientos en clase, los estudiantes utilizan las herramientas informáticas como fuente de referencia y apoyo para el aprendizaje que les permite incrementar su rendimiento académico (Venkatesh et al., 2016; Roshan, 2015; Romney, 2016). Las TIC juegan un papel indispensable en el proceso de aprendizaje por la facilidad de manejo existente puesto que superan las dificultades espacio-temporales, promueven ambientes cooperativos dentro de entornos digitales; al utilizar tecnologías de la información y la comunicación (TIC) se ofrecen entornos nuevos y atractivos de aprendizaje más efectivos y eficaces, que al contar con una gran cantidad de herramientas, permite a los alumnos ampliar los contenidos de las asignaturas, incrementar el nivel de desarrollo de identidad profesional, enriquecer y potenciar habilidades y competencias, que tienen impacto con la calidad del conocimiento adquirido y su aplicación en la vida profesional (Alzahrani, 2017 y Laaroussi et al., 2017).

\section{METOdOLOGÍA}

La metodología utilizada en esta investigación, es de tipo descriptivo y correlacional; fue realizada en el 2016 en la Ciudad de México dentro del Instituto Politécnico Nacional en la Unidad Profesional Interdisciplinaria de Ingeniería y Ciencias Sociales y Administrativas (UPIICSA) donde se imparten distintos programas educativos entre los que se encuentran: Administración Industrial; Ingeniería Industrial; Ingeniería Informática; Ciencias de la Informática; Ingeniería en Transporte.

\section{Participantes}

La población estudiantil de la unidad es de 13,000 alumnos aproximadamente, de la cual fue tomada una muestra representativa de 235 estudiantes de los distintos programas educativos de nivel licenciatura, para el tamaño de la muestra se utilizó el cálculo cuando se conoce el tamaño de la población, para esto se realizó con la fórmula de la ecuación 1.

$$
\mathrm{n}=\frac{\mathrm{N} * \mathrm{Z}_{\alpha}^{2} * \mathrm{p} * \mathrm{q}}{\mathrm{d}^{2}(\mathrm{~N}-1)+\left(\mathrm{Z}_{\alpha}^{2} * \mathrm{p} * \mathrm{q}\right)}
$$

\section{Donde:}

$\mathrm{N}=$ tamaño de la población

$Z$ = nivel de confianza,

$\mathrm{p}=$ probabilidad de éxito, o proporción esperada

$q=$ probabilidad de fracaso

$\mathrm{d}=$ precisión (Error máximo admisible en términos de proporción)

Para nuestro caso se utilizó un tamaño de población de 13000, con una seguridad del 95\%, una precisión del $3 \%$ y una proporción esperada del $5 \%$. Esto nos dice que se requieren encuestar a no menos de 73 estudiantes para poder tener una seguridad del 95\%, sin embargo, se logró encuestar a 235 alumnos.

Para la inclusión de los sujetos de estudio, se decidió realizar un muestreo aleatorio independiente del semestre o programa de estudios, esto a fin de que la información no fuera sesgada, en cuanto a la validez del instrumento se realizó una prueba de Cron Bach a un piloto de 7 estudiantes, el resultado fue un alfa de Cronbach (0.84). Asimismo, se analizó una correlación de Pearson entre las variables seleccionadas y las que se presentan en este estudio tienen una correlación positiva y alta. 


\section{Instrumento y Variables}

Para el desarrollo de la investigación fue utilizada una encuesta semiestructurada que consta de 28 preguntas divididas en 4 distintas secciones acerca del uso de las TIC entre las que se encuentran: actitud hacia las computadoras; uso de las TIC como entretenimiento; disponibilidad de TIC; uso de las TIC para la labor académica, de estas secciones, solamente se está estudiando el uso de TIC como entretenimiento y el uso de las TIC para la labor académica, las otras 2 secciones no se incluyen en este trabajo, debido a que su correlación con la variable dependiente (Internet como búsqueda de información para realizar tareas escolares) fue negativa.

Por consiguiente, se procedió a realizar un modelo para ver la relación que existe entre las variables independientes de uso de TIC (Computadora de casa, Diversión al utilizar la computadora, Videos como pasatiempo, Redes sociales para chatear, Ipads para hacer tareas, Computadora de la escuela y Redes sociales como medio de comunicación entre profesores y alumnos) dichas variables están relacionadas con aspectos académicos como búsqueda de información para hacer tareas y la variable dependiente (Búsqueda de Información en Internet para realizar tareas escolares). Se consideró la frecuencia de uso de las variables mencionadas en días de uso, a través de una escala del 0 al 7, donde 0 es nunca, 2 es 2 veces por semana, 3 es 3 veces por semana, 5 es 5 veces por semana y 7 es diario. Los datos obtenidos fueron analizados primero de forma descriptiva para observar la utilidad de la computadora para tareas escolares de acuerdo a los distintos programas de estudio que oferta la universidad de estudio. Después de analizo la utilidad del internet para tareas escolares por programa de estudio. Para finalizar se analizó la frecuencia de uso del internet para tareas escolares a la semana.

\section{Modelo}

Con los datos obtenidos se realizó un modelo de regresión lineal múltiple a través del método de mínimos cuadrados ordinarios, mismo que incluye las variables independientes y la variable dependiente, que se presentan en la figura 1. Asimismo, se presenta en la ecuación 2, las variables del modelo teniendo como variable dependiente la utilidad del internet para elaborar tareas y el resto son variables independientes. El modelo se estimó mediante mínimos ordinarios, con el procedimiento MCO de SAS (SAS Insitute Inc, 2002) versión 9.0. El análisis realizado para el modelo se enfocó en el uso de un estadístico denominado valor-p, el cual corresponde a la probabilidad de aceptar la hipótesis nula, comparada con el nivel de significancia $\alpha$ (se utilizó $\alpha=0,05)$.

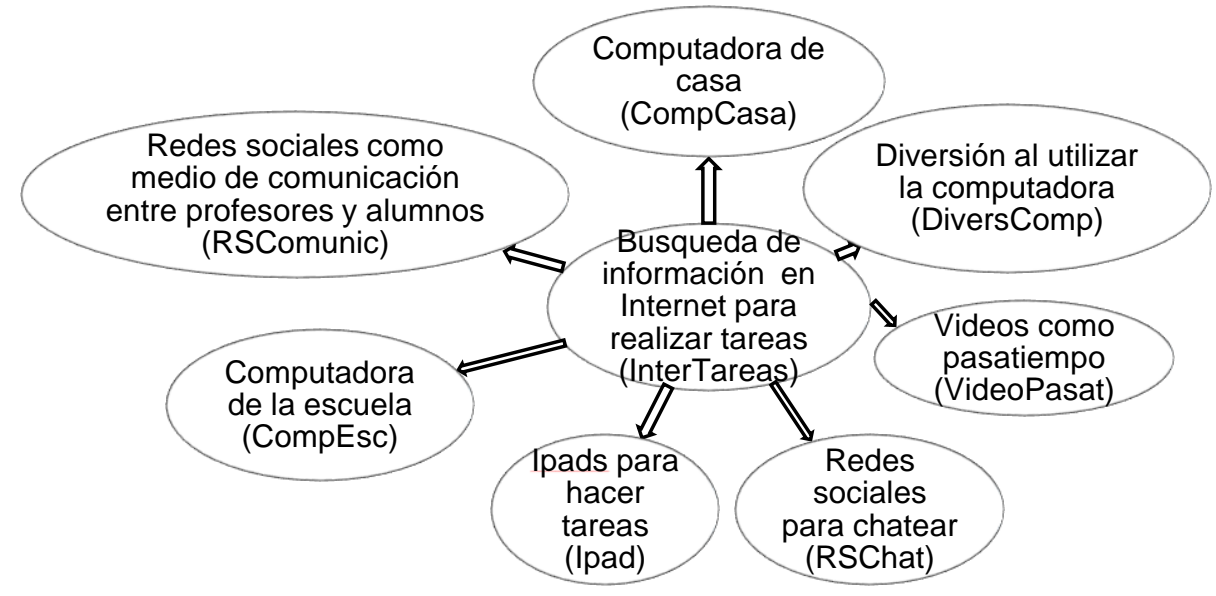

Fig. 1: variables que influyen en la búsqueda de información en internet para realizar tareas escolares en los estudiantes universitarios.

InterTar $=\beta_{0}+\beta_{1}$ CompCasa $+\beta_{2}$ DiversComp $+\beta_{3}$ VideoPasat $+\beta_{4}$ RSChat $+\beta_{5}$ Ipad + $\beta_{6}$ CompEsc $+\beta_{7}$ RSComunic

\section{RESULTADOS}

En esta sección se presenta en la Fig. 2 la utilidad de la computadora para tareas escolares, asimismo en la Fig. 3 se tiene la utilidad del internet de igual forma para actividades escolares, y se presenta en la Fig. 4 la frecuencia de uso en días que usan el internet a la semana por programa educativo para las tareas escolares, así mismo se presentan los resultados del modelo que contiene las variables que influyen el Internet como recurso de gran utilidad para la búsqueda de información para las tareas. 


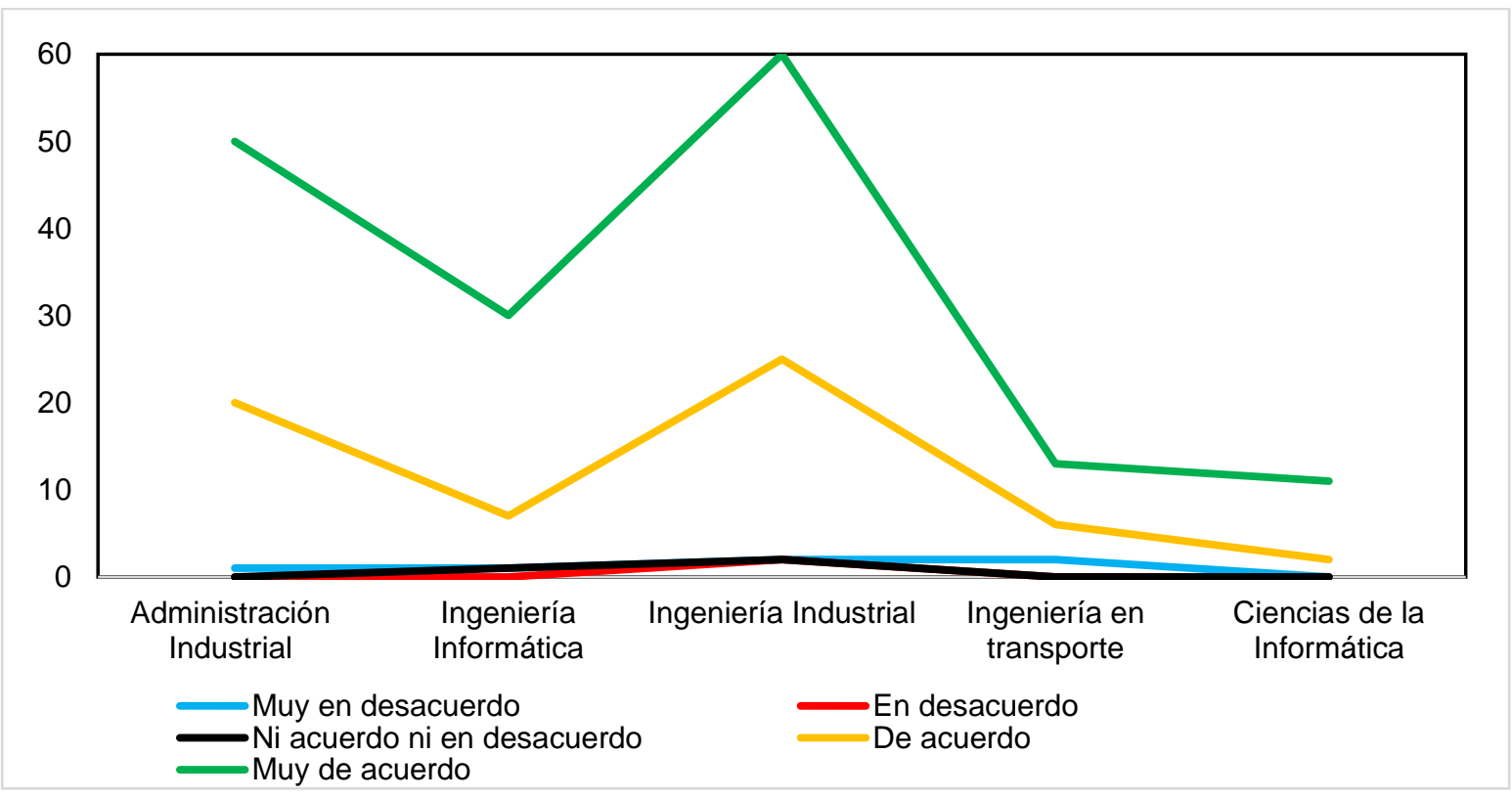

Fig. 2: Utilidad de la computadora para tareas escolares de acuerdo a los distintos programas educativos

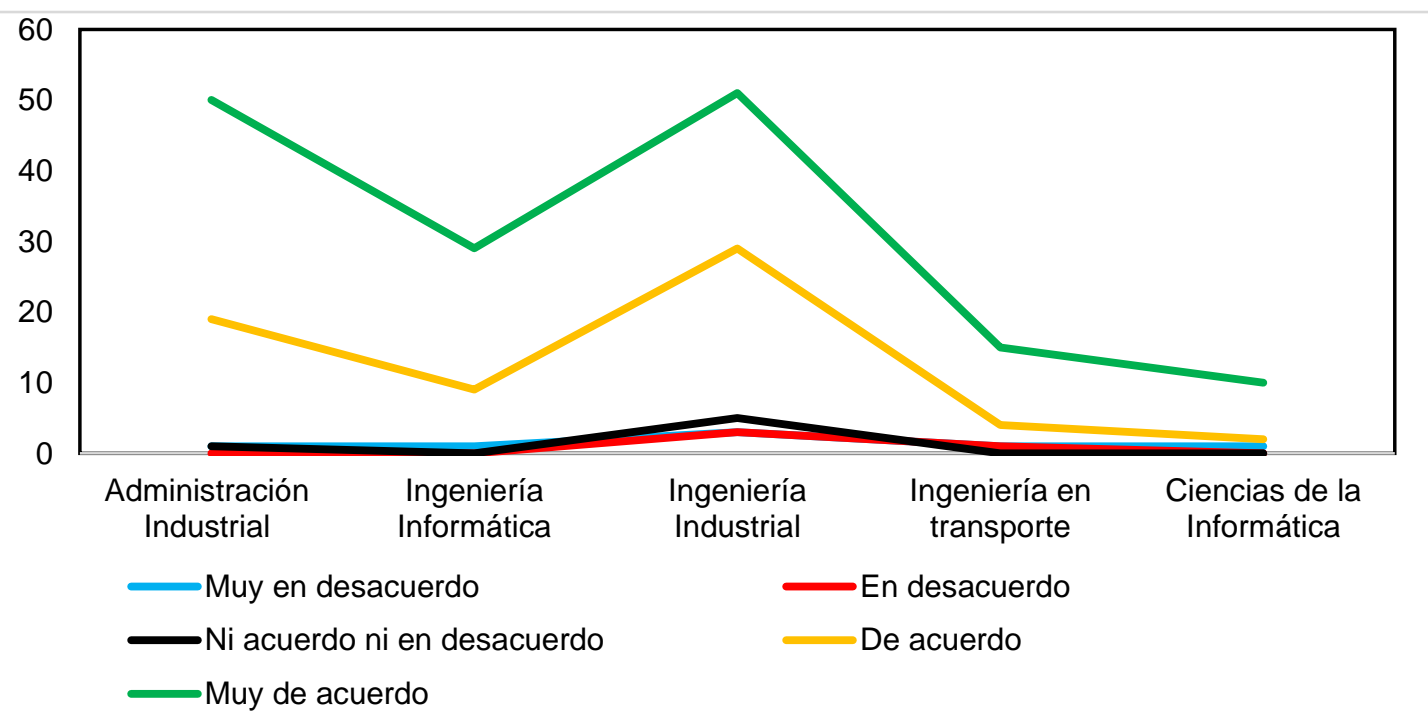

Fig. 3: Utilidad del internet para tareas escolares de acuerdo a los distintos programas educativos

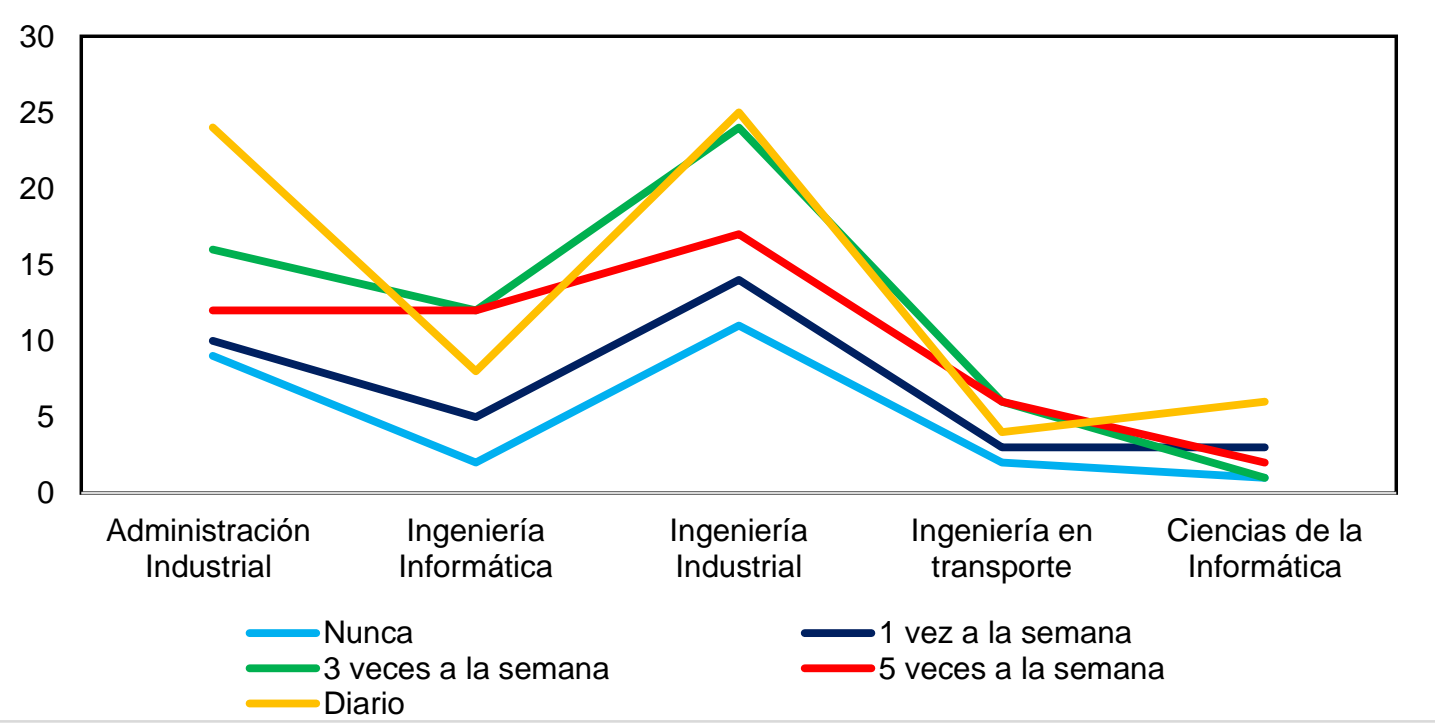

Fig. 4: Días que usan el internet para tareas escolares a la semana de acuerdo a los distintos programas educativos 
Tabla 1. Parámetros estimados por el método de mínimos cuadrados ordinarios, coeficientes de determinación y el error estándar.

\begin{tabular}{|c|c|c|c|c|c|}
\hline \multicolumn{6}{|c|}{ Variable dependiente: Búsqueda de Información en Internet para realizar tareas (InterTareas) } \\
\hline & Coeficiente & Desv. Típica & Estadístico $t$ & Valor $p$ & \\
\hline const & 0.9070 & 0.2564 & 3.5 & 0.0005 & *** \\
\hline Computadora de casa (CompCasa) & 0.6508 & 0.0582 & 11.1 & $<0.0001$ & *** \\
\hline $\begin{array}{l}\text { Diversión al utilizar la computadora } \\
\text { (DiversComp) }\end{array}$ & 0.2118 & 0.0483 & 43.8 & $<0.0001$ & $* * *$ \\
\hline Videos como pasatiempo (VideoPasat) & -0.0306 & 0.0185 & -16.5 & 0.0989 & * \\
\hline Redes sociales para chatear (RSChat) & 0.0321 & 0.0190 & 16.8 & 0.0936 & * \\
\hline Ipads para hacer tareas (Ipad) & 0.0331 & 0.0170 & 19.4 & 0.0534 & * \\
\hline Computadora de la escuela (CompEsc) & 0.0341 & 0.0168 & 20.2 & 0.0438 & ** \\
\hline $\begin{array}{l}\text { Redes sociales como medio de } \\
\text { comunicación entre profesores y } \\
\text { alumnos (RSComunic) }\end{array}$ & 0.0311 & 0.0195 & 15.9 & 0.0912 & * \\
\hline $\mathrm{R}^{2}$ & 0.7635 & & & \multirow{3}{*}{\multicolumn{2}{|c|}{$\begin{array}{l}*=p \leq 0.1 \\
* *=p \leq 0.05 \\
* * *=p \leq 0.01\end{array}$}} \\
\hline $\mathrm{R}_{\mathrm{a}}^{2}$ & 0.7495 & & & & \\
\hline Valor $\mathrm{p}$ (de F) & 5.34E-36 & & & & \\
\hline
\end{tabular}

Asimismo, se estimó el modelo que se presentó en una ecuación 2, y se presentan los resultados en la tabla 1 , relacionado con las variables que influyen en la variable dependiente (Búsqueda de Información en Internet para realizar tareas (InterTareas). Tomando en cuenta los resultados de los coeficientes obtenidos en la tabla 1; para el caso de la variable Utilidad de la computadora de casa para realizar tareas, esta tiene una influencia positiva en la variable dependiente: Internet como recurso de gran utilidad para la búsqueda de información para las tareas $(\beta=0.6508, p<0.01)$. De igual manera para la Diversión al utilizar la computadora para realizar tareas, también presenta una influencia positiva $(\beta=0.2118, p<0.01)$; no obstante los Videos como pasatiempo presentan una influencia negativa $(\beta=-0.0306, p<0.1)$. Aunado a esto la variable Redes sociales para chatear, también presenta una influencia positiva, en menor grado pues refleja un nivel de confianza del $91 \%$, pero se considera aun aceptable todavía $(\beta=0.0321, p<0.1)$; en el mismo caso se tiene la variable Ipads para hacer tareas $(\beta=0.0331, p<0.01)$. Al mismo se tiene la variable Uso de computadora de la escuela para tareas, misma que presenta una influencia positiva en la variable dependiente $(\beta=0.0341, p<0.05)$ al tener un $95 \%$ de nivel de confianza. Por último se tiene la variable Redes sociales como medio de comunicación $(\beta=0.0311, p<0.1)$ misma que influye de forma positiva en menor proporción; lo mismo sucede con los valores $R_{a}{ }^{2}(0.749)$, además de que los valores estadísticos $t$ son mayores o cercanos al valor 2 , y el valor $p$ de $F$ (5.34E-36), lo que permite validar el modelo de regresión.

En consecuencia, se puede asegurar que los valores estimados de la regresión previamente obtenidos en el modelo influyen en la Búsqueda de Información en Internet para realizar tareas. En la ecuación 3 se puede observar que las redes sociales, los equipos de cómputo de las escuelas y la comunicación entre alumnos y profesores por medio de redes sociales influyen positivamente en el internet como herramienta para la búsqueda de información de tareas escolares, sin embargo, se observa también que los videos como pasatiempo influyen de forma negativa en la realización de tareas escolares.

InterTareas $=0.9070+0.6508$ CompCasa +0.2118 DiversComp -0.0306 VideoPasat + 0.0321 RSChat +0.0331 Ipad +0.0341 CompEsc +0.0311 RSComunic

\section{DISCUSIÓN}

Debido al incremento que ha tenido el internet desde su inclusión en la sociedad, este se ha convertido en un recurso indispensable para los estudiantes de educación superior, y esta investigación refuerza el hecho anterior. En el estudio realizado se puede observar que los alumnos consideran de gran utilidad las Computadoras Personales (PC) con acceso a internet para la realización de tareas escolares, sin embargo, contrastando los hallazgos de Linne (2015); gran parte de los estudiantes perteneciente a este nivel educativo (70\%), consideran que el acceso a internet es una manera de crear distractores cuando se realizan las tareas escolares. Aunque Line (2015) solo presenta porcentajes de forma descriptiva y esta investigación muestra coeficientes que demuestran el incremento de búsqueda de información en internet a través de las computadoras, lo cual hace esta investigación confiable, aunque se considera de gran apoyo lo sustentado por Line (2015). 
En la fig. 3 se puede percibir que los estudiantes de los distintos programas académicos que ofrece la universidad de estudio, están de acuerdo que el internet es una herramienta de gran utilidad para realizar actividades académicas, y se tiene acceso de acuerdo con la SCT (2017) en distintos puntos públicos a lo largo del país, asimismo Khan y Markauskaite (2017) y Linne (2015) reiteran la importancia de su uso que conlleva a la creación de aprendizajes competitivos a través de los recursos a los que se tiene acceso, además se incrementa la comunicación entre profesores y alumnos y da paso a la consulta de diversas fuentes que permiten la autonomía en el proceso de aprendizaje así como su fortalecimiento.

En la fig. 4, se pueden observar que los resultados obtenidos complementan a los descritos anteriormente, puesto que puede apreciarse que la mayoría de los estudiantes utilizan el internet por lo menos una vez a la semana con fines académicos, creando así espacios digitales dentro de la educación superior e incrementando el uso de las TIC, que es confirmado por Pérez y Pino (2017), Basantes et al. (2017) y Casillas, et al. (2016) quienes opinan que la implementación de las TIC en los procesos de enseñanza y aprendizaje crea nuevos entornos para desarrollar habilidades a través de distintos dispositivos, que despierta la inquietud por explorar e implementar su uso en las actividades escolares.

Así mismo y tomando en consideración el modelo presentado de forma numérica en la ecuación 2, se puede apreciar que efectivamente las TIC han ido incursionando en el ámbito educativo, siendo hoy en día, herramientas de gran utilidad para los estudiantes, tal es el caso del internet, dada a la gran cantidad de información a la que se tiene acceso, los alumnos universitarios ocupan dicha herramienta para la búsqueda de datos específicos para la realización de tareas y proyectos escolares, influyendo así en la creación de nuevos conocimientos; la autonomía del proceso de aprendizaje; la calidad de la educación; el desarrollo de nuevas habilidades específicas, lo cual se ve reflejado en el aumento considerable del rendimiento académico de los estudiantes. Lo cual coincide con Roshan (2015) pues indicó que el internet en conjunto con las TIC aumenta el promedio de los alumnos en al menos un 4\% trimestralmente (Roshan, 2015). Lo cual es un buen punto para dar continuidad a esta investigación y realizar otro modelo para medir el impacto en el rendimiento académico.

En cuanto a la comunicación de los alumnos por medio de las TIC, esta investigación presenta un estimado de 0.0311 , por otro lado, la investigación de Venkatesh et al. (2016) argumenta un estimado de 0.03 sobre el uso de TIC para comunicación en 12 universidades de Quebec. Lo cual implica que en México se está teniendo un aspecto muy similar al de Canadá en cuanto a la percepción de los alumnos sobre las TIC para comunicación a pesar de que las condiciones socioeconómicas son muy diferentes.

Las vías de acción para el mejor empleo de las TIC dentro del nivel superior son fomentar el uso de la computadora tano en el hogar como en la escuela, para realizar tareas; además los docentes deben de generar contenidos educativos divertidos, es decir a través de juegos virtuales que tengan tiempo de respuesta y que incluyan material multimedia que estimule el compromiso y la lealtad a los juegos para que los estudiantes pasen más tiempo en el juego y refuercen su aprendizaje; los profesores también deben de crear video tutoriales como pasatiempo para los alumnos, para que los videos no tengan un impacto negativo en la educación. En México todos los alumnos hacen uso de las redes sociales, por lo cual los docentes también deben de hacer estrategias de juegos educativos que refuercen temas complejos y que ellos puedan chatear para comunicarse al hacer trabajos en equipo.

\section{CONCLUSIONES}

El internet es un recurso útil para buscar información y para elaborar tareas académicas en el nivel superior, en esta investigación se encontró los factores que contribuyen al uso del internet para las tareas son: el uso de la computadora del hogar, la forma divertida de usar la computadora, así como el uso de las redes sociales para chatear, por otro lado, también el hacer tareas en un Ipad, así como el uso de la computadora de la escuela y las redes sociales como medio de comunicación. No obstante, los Videos como pasatiempo presentan una influencia negativa en el internet como recurso de utilidad para buscar información y hacer tareas. Por lo cual se sugiere hacer un buen uso de la tecnología, pues en el caso de los videos como pasatiempo se genera una distracción, en consecuencia, los docentes deben crear videos educativos para sus estudiantes y que incluyan también pasatiempo.

\section{AGRADECIMIENTOS}

Agradecemos las facilidades otorgadas para el desarrollo del presente trabajo al Instituto Politécnico NacionalUnidad Profesional Interdisciplinaria de Ingeniería y Ciencias Sociales y Administrativas. Proyecto SIP 20161046 Las habilidades informáticas del capital humano como fuente de crecimiento económico. 


\section{REFERENCIAS}

Alzahrani, M. G., The Developments of ICT and the Need for Blended Learning in Saudi Arabia, Journal of Education and Practice, 8(9), 79-87 (2017)

Anshari, M., Alas, Y., y L. S. Guan, Pervasive knowledge, social networks, and cloud computing: E-learning 2.0, doi: 10.12973/eurasia.2015.1360a, EURASIA, J. Math., Sci Tech. Ed., 11(5), 909-921 (2015)

Ausín, V., V Abella, V. Delgado y D. Hortigüela, Aprendizaje Basado en Proyectos a través de las TIC: Una Experiencia de Innovación Docente desde las Aulas Universitarias, doi:10.4067/S0718-50062016000300005, Formación Universitaria, 9(3), 31-38 (2016)

Basantes, A. V., M. E. Naranjo, M. C. Gallegos y N. M. Benítez, Los Dispositivos Móviles en el Proceso de Aprendizaje de la Facultad de Educación Ciencia y Tecnología de la Universidad Técnica del Norte de Ecuador, doi:/10.4067/S0718 50062017000200009, Formación Universitaria, 10(2), 79-88 (2017)

Cámara de Diputados, Constitución Política de los Estados Unidos Mexicanos, México (2017)

Caputi, V. y A. Garrido, Student-oriented planning of e-learning contents for Moodle, doi: 10.1016/j.jnca.2015.04.001, J. Netw. Comput. Appl., 53, 115-127 (2015)

Casillas-Alvarado, M.A., A. Ramírez Martinel y J.C. Ortega Guerrero, Afinidad tecnológica de los estudiantes universitarios, Innovación Educativa, (México, D.F.), 16(70), 151-175 (2016)

Eid, M.I. y Al-Jabri, I.M., Social Networking, knowledge sharing, and student learning: The case of university students, doi:10.1016/j.compedu.2016.04.007, Comput. Educ., 99, 14-27 (2016)

Fainholc, B., De cómo las TICs podrían colaborar en la innovación socio-tecnológico-educativa en la formación superior y universitaria presencial, RIED, Revista iberoamericana de educación a distancia, 11(1) (2008)

Faúndez, C. A., A. A. Bravo, G. P. Ramírez y H. F. Astudillo, Tecnologías de la Información y la Comunicación (TIC) en el Proceso de Enseñanza-Aprendizaje de Conceptos de Termodinámica como Herramienta para Futuros Docentes, doi:10.4067/S0718-50062017000400005, Formación Universitaria, 10(4), 43-54 (2017)

Federal, P. E., Plan Nacional de Desarrollo 2013-2018, Ciudad de México, México (2013)

Flavin, M., Free, Simple and Easy to Use: Disruptive Technologies, Disruptive Innovation and Technology Enhanced Learning, doi.org/10.1057/978-1-137-57284-4_2,19-52, In Disruptive Technology Enhanced Learning, Palgrave Macmillan, Londres, Inglaterra (2017)

Garcés-Suárez, E., E. Garcés Suárez y O. Alcívar Fajardo, Las tecnologías de la información en el cambio de la educación superior en el siglo XXI: reflexiones para la práctica, Revista Universidad y Sociedad, 8(4), 171-177 (2016)

Khan, M.S.H. y L. Markauskaite, Approaches to ICT-enhanced teaching in technical and vocational education: a phenomenographic perspective, doi:10.1007/s10734-016-9990-2, Higher Educ, 73(5), 691-707 (2017)

Laaroussi, A., S. Ajana, S. Bakkali, K. Faraj, y O. Cherkaoui, E-learning Foresight for Renewable Energy Technology in Higher Education in Morocco, https://doi.org/10.1007/978-3-319-46568-5_2, In Europe and MENA Cooperation Advances in Information and Communication Technologies Springer International Publishing, (530) 13-23 (2017)

Linne, J., Estudiar en Internet 2.0.: Prácticas de jóvenes universitarios de la Ciudad de Buenos Aires, Comunicación y sociedad, (23), 195-213 (2015)

Manca, S. y M. Ranieri, Facebook and the others. Potentials and obstacles of social media for teaching in higher education, doi:10.1016/j.compedu.2016.01.012, Comput. Educ., 95, 216-230 (2016)

Pérez, O. G. B. y J. W. F. Pino, Rol de la gestión educativa estratégica en la gestión del conocimiento, la ciencia, la tecnología y la innovación en la educación superior, doi: 10.1016/j.edumed.2016.12.001, Educación Médica (2017)

Prieto-Velasco, J.A. y A. Fuentes-Luque, A collaborative multimodal working environment for the development of instrumental and professional competences of student translators: an innovative teaching experience, doi:10.1080/1750399X.2016.1154344, The Interpreter and Translator Trainer, 10(1), 76-91 (2016)

Pruet, P., C. S. Ang y D. Farzin, Understanding tablet computer usage among primary school students in underdeveloped areas: Students' technology experience, learning styles and attitudes, doi:10.1016/j.chb.2014.09.063, Comput. Hum. Behav, 55, 1131-1144 (2016)

Romney, C. A., Impact of Undergraduate Tablet PC Use on Retention in STEM Majors, doi.org/10.1007/978-3-319-311937_20, In Revolutionizing Education with Digital Ink. Springer International Publishing, 301-305 (2016)

Roshan, S., The flipped classroom: touch enabled, academically proven, doi:10.1007/978-3-319-15594-4_21, The Impact of Pen and Touch Technology on Education, 215-222 (2015)

Secretaría de Comunicaciones y Transporte, Presenta SCT Programa de Conectividad Digital para consolidad los avances de la Reforma de Telecomunicaciones, Ciudad de México (2017)

Thomas, M. A., Y. Li y T. Oliveira, Nuances of development contexts for ICT4D research in least developed countries: An empirical investigation in Haiti, doi:10.1016/j.tele2017.05.001, Telemat Informat, 34(7), 1093-1112 (2017) 
Trust, T., Motivation, Empowerment, and Innovation: Teachers' Beliefs about How Participating in the Edmodo Math Subject Community Shapes Teaching and Learning, doi: 10.1080/15391523.2017.1291317, J. of Research on Technology in Education, 49(1-2), 16-30 (2017)

U. N. I. C. E. F. y F. D. B. Arceo, Las políticas TIC en los sistemas educativos de América Latina: Caso México, 1aㅡ Ed., 5, 9-111, UNICEF, Argentina (2013)

UNESCO, Políticas TIC en los sistemas educativos de América Latina. Informe sobre tendencias sociales y educativas en América Latina 2014, Buenos Aires (2014)

Vahtivuori-Hänninen, S. y H. Kynäslahti, Icts in a School's Everyday Life-Developing the Educational Use of Icts in Finnish Schools of the Future, doi:10.1007/978-94-6300-776-4_16, Miracle of Education, 241-252 (2016)

Venkatesh, V., J. Rabah, M. Fusaro, A. Couture, W. Varela y K. Alexander, Factors impacting university instructors' and students' perceptions of course effectiveness and technology integration in the age of Web 2.0, doi:10.7202/1037358ar, McGill Journal of Education/Revue des sciences de l'éducation de McGill, 51(1), 533-561 (2016)

Vicent, L., S. Villagrasa, D. Fonseca y E. Redondo, Virtual learning scenarios for qualitative assessment in higher education 3D arts, doi: 10.3217/jucs-021-08-1086, J. of Universal Computer Science, 21(8), 1086-1105 (2015)

Witteveen, L., R. Lie, M. Goris y V. Ingram, Design and development of a digital farmer field school. Experiences with a digital learning environment for cocoa production and certification in Sierra Leone, doi:10.1016/j.tele.2017.07.013, Telemat. Informat., 34(8), 1673-1684 (2017)

Yamashita, K. y H. Yasueda, Project-based learning in out-of-class activities: flipped learning based on communities created in real and virtual spaces, doi:10.1016/j.procs.2017.08.108, Procedia Computer Science, 112, 1044-1053 (2017) 